\title{
CORRIGENDUM
}

\section{Immediate early gene X-1 (IEX-1) is differentially induced by retinoic acids in NB4 and KG1 cells: possible implication in the distinct phenotype of retinoic acid-responsive and -resistant leukemic cells}

\author{
A Arlt, J Minkenberg, B Kocs, M Großmann, M-L Kruse, UR Fölsch and H Schäfer
}

Leukemia (2006) 20, 1651. doi:10.1038/sj.leu.2404347

Correction to: Leukemia (2004) 18, 1646-1655.

doi:10.1038/sj.leu.2403481

In the paper entitled 'The expression of immediate early gene X-1 (IEX-1) is differentially induced by retinoic acids in NB4 and KG1 cells: possible implication in the distinct phenotype of retinoic acid-responsive and -resistant leukemic cells' we inadvertently assigned KG1 leukemic cells to a $t(11 ; 17)$ karyotype expressing the PLZF-RAR fusion protein. In fact, KG-1 cells do not express PLZF- and PML-RAR fusion proteins. Whatsoever, the findings on the differential induction of IEX-1 by retinoic acids in NB4 and KG1 cells are not affected by this mistake, because the distinct induction kinetics of IEX-1 found in these two cell lines relate to their distinct responsiveness in terms of proliferation, apoptosis and differentiation which is determined by PML-RAR (NB4) and its wild-type counterparts (KG1). Hence, retinoic acid induced IEX-1 expression in PML-RAR is transient and does not confer higher apoptotic sensitivity as observed in KG1 cells that persistently express IEX-1 upon retinoic acid treatment and become highly sensitive to apoptotic stimuli.

The authors apologize for these errors. 